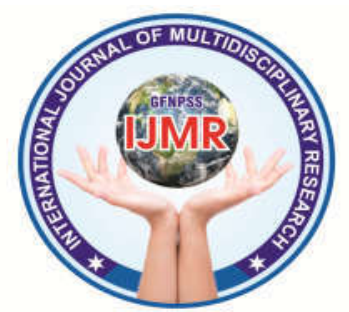

ISSN (0): 2582-693X

Original Article

\title{
PIEZOELECTRIC VS CONVENTIONAL ROTARY TECHNIQUE FOR MANDIBULAR IMPACTED THIRD MOLAR SURGERY
}

\author{
DR AMIT KUMAR SHARMA ${ }^{1}$ DR AKSHAT GUPTA ${ }^{2}$ DR HEMANG PABARI ${ }^{3}$ DR \\ SANCHAYEENI K PATHAK ${ }^{4}$ DR CHETNA GABHANE ${ }^{5}$ DR NILESH ODEDRA ${ }^{6}$ \\ ${ }^{1}$ HOD, Daswani Dental College and Research Centre, Kota \\ ${ }^{2}$ Reader, Daswani Dental College and Research Centre, Kota \\ ${ }^{3}$ PG Trainee, Daswani Dental College and Research Centre, Kota \\ ${ }^{4}$ Senior Lecturer, Daswani Dental College and Research Centre, Kota \\ ${ }^{5,6}$ PG Trainee, Daswani Dental College and Research Centre, Kota
}

Corresponding Email: bala.dr0359@gmail.com, aksgupta18@yahoo.com, hemang.pabari12@,gmail.com, sanchu12108@,gmail.com, chetnagabhane22@gmail.com, nileshodedra@gmail.com

\section{ABSTRACT}

Purpose: The aim of this study to evaluate and compare the operative and post-operative outcomes of the mandibular impacted third molar removal using piezoelectric technique and conventional rotary bur technique.

Methods: 30 patients, with age of 18-40 years, with asymptomatic bilateral impacted mandibular third molars, were selected for the study and randomly surgical extraction were done either using piezoelectric or the conventional rotary bur technique on each site. The intraoperative time taken, post-operative pain, swelling, and trismus were assessed over a follow-up period of 7 days postoperatively. Result: Study and control group were compared using paired $\mathrm{t}$-test. There was statistical significance in reduction of pain and swelling in study group, where the duration of surgery was increased in study group.

Conclusion: Even if Piezoelectric technique takes more time, but can be used safely as another technique to conventional surgical method with surgical bur and handpiece for removal of impacted mandibular third molars.

Keyword: Impaction, Rotary, Piezosurgery, Third molar, Osteotomy. 


\section{INTRODUCTION}

Impacted molars square measure often encountered in clinical work, with a prevalence of $30 \%$ to $60 \%[1-4]$. It's been well documented that wedged third molars, either partial or complete, are related to many complications together with pericoronitis, regional pain, odontogenic abscesses, trismus, distal tooth decay, cysts, tumors, and crowding [5-8] . So, symptomatic or asymptomatic impacted third molars are often extracted to cut back the abovementioned clinical symptoms. Conventional osteotomies for surgical removal of impacted third molars involved the use of chisel-mallet, rotatory surgical burs and oscillating saws. The split bone technique was introduced by ward in 1956[9] chisels were the preferred instruments for bone removal. But another study[10]stated that the technique was more painful and most of the patients may not tolerate the jarring effect of chisel and mallet while operating under local anaesthesia. Hence, it was preferable to opt for rotary instruments for bone removal and tooth sectioning for surgery under local anaesthesia but Use of rotatory osteotomy and oscillating saws result in irregular surfaces and marginal osteonecrosis due to the high temperature generated during osteotomy[11] . In 1998, Torrella et al. published a technical note describing a bone window osteotomy to lift the maxillary sinus with an ultrasonic piezoelectric ablator. After testing this technique also for surgery on the maxillary sinus, Tomaso Vercellotti understood its benefits and took note of its drawbacks.

Piezotome ultrasonic generator is a new system for cutting bone with micro vibration. These are created by the piezoelectric effect: certain ceramics and crystals deform when an electric current is passed across them, resulting in oscillations of ultrasonic frequency. This is three times more powerful than normal ultrasound and therefore, can cut highly mineralized bone. The piezoelectric ultrasonic vibrations $(25-30 \mathrm{khz})$ which generate micrometric vibration ranging from $(60-200 \mathrm{~mm} / \mathrm{sec})$, because of its micrometric and selective cut, the piezoelectric device produces safe and precise osteotomies without any osteonecrosis damage. The main advantage of the piezosurgery is its selective cut that recognizes tissue hardness and works only on mineralized structures. In dentistry, this device is 


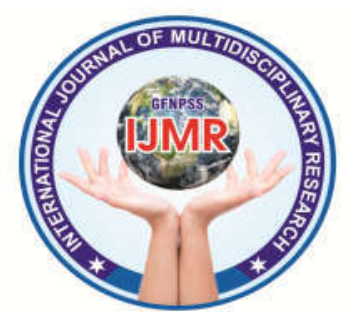

suggested to find clinical applications in by same operator; the time between both periodontal surgery, sinus grafting, was two weeks.

intraoral gaining of bone chips, teeth

Patients were subdivided into two extraction, alveolar crest expansion 26-31 for distraction osteogenesis and orthognathic, preprosthetic surgeries etc.

Therefore, in this study, we carried out an mean clinical trial to compare piezosurgery with conventional rotary osteotomy techniques, with notice of duration of procedure and postoperative fall out including pain, swelling, and trismus.

\section{MATERIALSANDMETHODS}

After procuring the ethical approval from the ethical committee. 30 patients were selected conveniently, fulfilling the inclusion criteria from the outpatient clinic of Oral \& Maxillofacial Surgery Department, Mahatma Gandhi Dental College, Jaipur. Participants required removal of their bilateral impacted mandibular third molars were selected irrespective of sex, caste and socio- economic status. The study was conducted in our institution between 1 st January 2018 to 1 st January 2019.

Because this is a split mouth study design, each patient underwent two operations for removal of their bilateral impacted mandibular third molars operated 


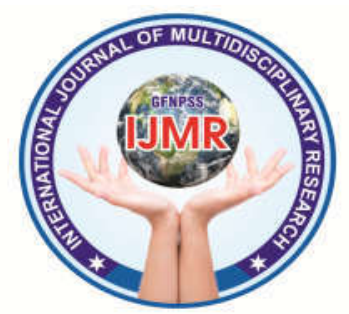

- Patients with any pathological conditions related to the site of surgery.

- Patients with infection around the lower third molar.

- Heavy smokers.

- Pregnancy or lactation.

\section{Materials}

○ W\&H Piezomed surgical unit.

○ Micro motor surgical unit with straight handpiece \& Carbide surgical burs for osteotomy.

\section{METHODOLOGY}

Pre-operative evaluation of patient was done which include, proper case history, opening of mouth was evaluated with Vernier calliper, facial measurement was obtained with thread as baseline measurements for assessment of edema, Radiographic examination and Routine blood investigations were done .

The surgical procedure was carried out in an operating room under strict aseptic conditions. The surgeries were performed under local anaesthesia (lignocaine 2\% with epinephrine 1:200000). The nerve blocks used were inferior alveolar, lingual and long buccal. For both sides, Standard Ward's Incision was given. After flap reflection in study group (S) bone 


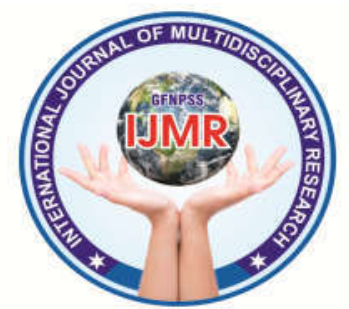

was continued in a similar manner as the study side. Followed by Proper debridement of the socket and flap closure
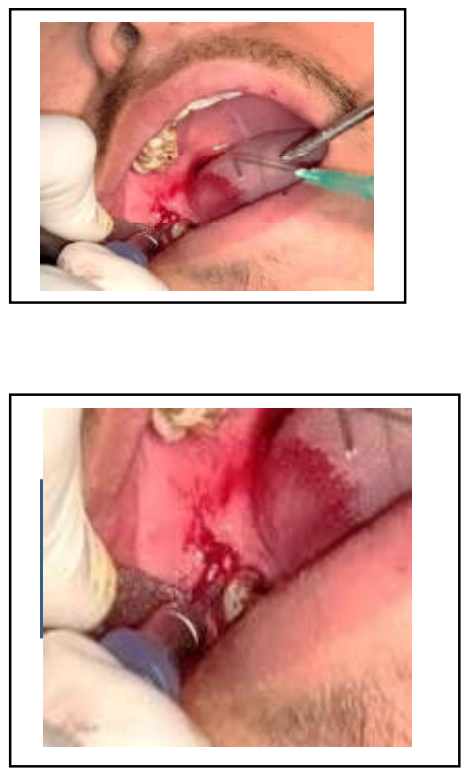

Figure 2: Bone guttering with micro motor and carbide bur All patients were given postoperative instructions following extractions. Antibiotics and Analgesics postoperatively. Assessment of patients was done at $3 \mathrm{rd}$ and 7 th postoperative days based on the following parameters:

1. Duration of surgery

2. Pain Visual Analogue Scale(VAS)

3. Swelling

4. Mouth Opening

\section{STATISTICAL ANALYSIS}

The variables included in the study were tested for normality using Kolmogrov Smirnov test. Because the data were randomly distributed, Study and
Control groups were compared regarding the study variables using paired t- test. Initial values were compared in the same group, using paired t test. Significance level was set at the 5\% level. Statistical analysis was performed, using SPSS version 21.0.

\section{RESULTS}

Duration of surgery was recorded in both the groups from the incision till the suturing.. Significantly more time was required in the study than in the control group (mean $=30.76$ and 19.60, $\mathrm{P}$

The mean post-operative pain after 1, 3 and 7 days, mean VAS scores in the study group was lower than that in the control group (mean in study= 3.56, 2.03 and 0.43 compared to mean in control $=2.8$, 5.66 and 2.56). The change from 1 to 7 days was statistically significant in the study and control group $\mathrm{P}<0.0001$ for both (Table 2).

Regarding postoperative swelling Significant differences existed between mean measurements indicating swelling after 3 and 7 days. On 3rd and 7th day swelling was greater at $(\mathrm{C})$ than an $(\mathrm{S})$ (figure 3)

Maximum mouth opening between groups at different follow up periods. Significant differences calculated between 


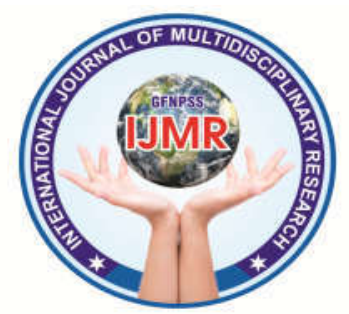

mean measurements, indicating mouth

Figure 3. Comparison of swelling between (S)

opening at baseline, after 3 and 7 days $(\mathrm{P}=$ and $(\mathrm{C})$ at different follow up periods

$<0.0001,<0.0001,<0.0001)$. At baseline, after 3 and 7 days, mouth opening in the (C) sides was less than at (S) sides. The change from baseline to 7 days was statistically significant in the $(\mathrm{S})$ or the $(\mathrm{C})$ group $(\mathrm{P}<0.0001)$ (Table3).

Table 1: Comparison of time taken for Operation between study and control

\begin{tabular}{|l|l|l|}
\hline & Study & Control \\
\hline Mean(SD) & $30.76(5.23)$ & $19.60(2.66)$ \\
\hline $\begin{array}{l}\text { Pvalueofpaired } \\
t \\
\text { test }\end{array}$ & $<0.0001^{*}$ & \\
\hline
\end{tabular}

*Statistically significant at $\mathrm{P} \leq 0.0001$

Table2: pain(VAS) at different interval of time periods

\begin{tabular}{|l|l|l|l|}
\hline & \multicolumn{2}{|l|}{ Mean(SD) } & $\begin{array}{l}\text { P value of } \\
\text { paired t } \\
\text { Test }\end{array}$ \\
\cline { 2 - 4 } & Study & Control & \\
\hline After 3 days & $2.03(0.85)$ & $5.66(1.24)$ & $<0.0001^{*}$ \\
\hline After 7 days & $0.43(0.62)$ & $2.56(0.67)$ & $<0.0001^{*}$ \\
\hline $\begin{array}{l}\text { P value of } \\
\text { paired t } \\
\text { test }\end{array}$ & $<0.0001^{*}$ & $<0.0001^{*}$ & \\
\hline
\end{tabular}

*: Statistically significant at $\mathrm{P} \leq 0.0001$ 


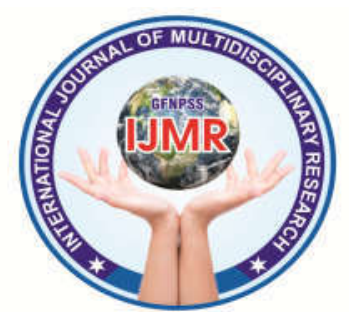

recurrent pericoronitis, cellulitis, abscess, cysts and tumors, unrestorable caries, periodontal breakdown, and prophylactic removal[13] . However, surgery is frequently associated with many possible complications, such as postoperative pain, swelling and trismus. Less commonly observed complications include infection, dry socket, trigeminal nerve injuries, and rarely, fracture of the mandible. (Mantovani et al.[14]. Recently, research and the application of advanced principles Piezosurgery or the application of piezoelectric, ultrasonic vibrations to make precise and safe osteotomies. To improve quality of life for patients, this work was carried out to evaluate the efficiency of piezosurgery vs. conventional surgical technique in removal of the impacted mandibular third molar and postoperative complications after the mandibular third molar removal.

\section{Surgical time}

Surgical extraction of impacted mandibular third molar is one of the most common oral surgical procedures. The indications for its removal include recurrent pericoronitis, cellulitis, abscess, cysts and tumors, unrestorable caries, periodontal breakdown, and prophylactic removal[13] . However, surgery is frequently associated with many possible complications, such as postoperative pain, swelling and trismus. Less commonly observed complications include infection, dry socket, trigeminal nerve injuries, and rarely, fracture of the mandible. (Mantovani et al.[14]. Recently, research and the application of advanced principles Piezosurgery or the application of piezoelectric, ultrasonic vibrations to make precise and safe osteotomies. To improve quality of life for patients, this work was carried out to evaluate the efficiency of piezosurgery vs. conventional surgical technique in removal of the impacted mandibular third molar and postoperative complications after the mandibular third molar removal.

\section{PAIN,SWELLING AND TRISMUS}

Pain was evaluated using the visual analogue scale (VAS). The present study showed that there was a significant statistical difference between the study group and the control group from the second postoperative day till the seventh. Whereas the VAS mean score decreased in study group from 2.03 on the third day after surgery to 0.43 on the seventh day, there was a reduction in VAS mean score from 5.66 on the third postoperative day to 2.56 on the seventh in the control group. 


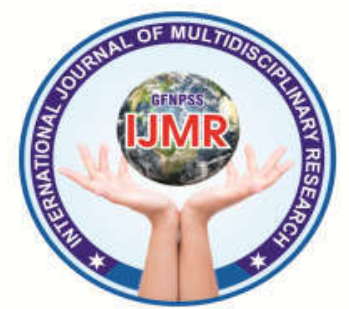

These results run parallel to Barone et al., Goyal et al., Piersanti et al., Arakji et al. where the authors tested the efficiency of piezosurgery versus the conventional technique in regards of pain using the same technique as this present work, namely the VAS. They also used another method for evaluating pain by recording the amount of analgesics taken after surgery, and concluded that there is significant difference between the two groups [17, 21-23]. Also, Mantovani et al [14] conducted a similar study and evaluated pain. They concluded that the mean pain evaluation reported by patients who underwent tooth removal using piezosurgery was significantly lower than that reported after bur bone removal, reaching statistical difference after 4 days. In all cases, they reported postoperative pain evaluated on the VAS was greatest on the day of surgery and then decreased progressively daily until day 6 after surgery.

Furthermore, swelling and trismus were evaluated in our study. Trismus measures were recorded before the surgery, on the third day, and seventh days. Regarding facial edema it was calculated by taking the mean of sum of three measurements:(the distance between the lateral corner of the eye and the angle of the mandible; the distance between tragus and the outer corner of the mouth; and the distance between tragus and soft tissue pogonion. It was taken in account the mean of sum of these measures); It was found that there is a significant difference between piezosurgery group and conventional handpiece and surgical bur technique, and that the mean facial swelling in study group varied from the third day after surgery to the seventh day from $12.67 \mathrm{~cm}$ to $12.58 \mathrm{~cm}$, whereas it varied from $12.8 \mathrm{~cm}$ to $13.23 \mathrm{~cm}$ in the control group.

Pappalard et al. [24] performed a comparative study between piezosurgery and conventional rotary surgery, and deduced that swelling was significantly less in the piezosurgery group. Meanwhile, trismus was assessed. Results have shown a significant difference between the two groups: where the range of mouth opening varied from the baseline to the seventh day, from $42.36 \mathrm{~mm}$ to $47.7 \mathrm{~mm}$ in the study group, whereas he values recorded in the control group varied from $42.43 \mathrm{~mm}$ to $36.68 \mathrm{~mm}$. These findings are similar to a study conducted by Sortino et al.[15] on 100 patients, 50 of whom were treated by the conventional method using the surgical 


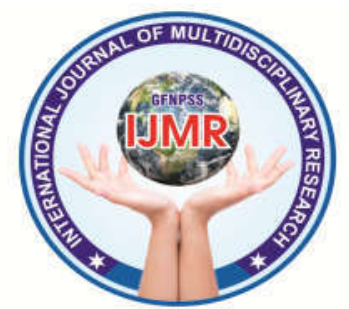

bur and 50 using the piezosurgery. Mozzati et al. [25] have shown parallel results with this study, they conducted a study on 15 patients. It was observed that the range of mouth opening increased more significantly in their study group than in the control group. These results run parallel to the current study results. Moreover, Goyal et al.[17] while conducting a similar study on 40 patients, their study has shown that despite extended operating time, the trismus was significantly lower in the piezotome group. In one Meta-analysis study showed that, although patients undergoing piezosurgery experienced longer surgery time, they had less postoperative swelling, indicating that piezosurgery is a promising alternative technique for extraction of impacted third molars. [26]

\section{CONCLUSION}

Within the limitation of this study, it will be concluded that:

Piezosurgery technique can be used as another technique to conventional surgical method with surgical bur and handpiece for removal of impacted mandibular third molars. It offers better healing, compartively reducing pain, range of mouth opening and postoperative edema. The most drawback was the time of surgery since the surgical bur and handpiece were faster in the surgical time. Yet, increasing the operator's experience could diminish this gap. Although piezosurgery is fast being adopted for major surgical procedures, its cost remains one in every of the foremost reasons for its continued limited usage in minor oral surgery like impacted third molar removal. At the top piezosurgery is way better than conventional rotary technique but furthermore studies are needed with larger sample size and longer follow up period for better evaluation and more conclusive results.

\section{REFERENCES}

01. Waite PD, Reynolds RR. Surgical management of impacted third molars. Semin Orthod 1998; 4:113123.

02. Breik O, Grubor D. The incidence of mandibular third molar impactions in different skeletal face types. Aust Dent J 2008; 53:320-324.

03. Celikoglu M, Miloglu O, Kazanci F. Frequency of agenesis, impaction, angulation, and related pathologic changes of third molar teeth in orthodontic patients. J Oral Maxillofac Surg 2010; 68:990-995.

04. Kumar BS, TS MV, Raman U. To 


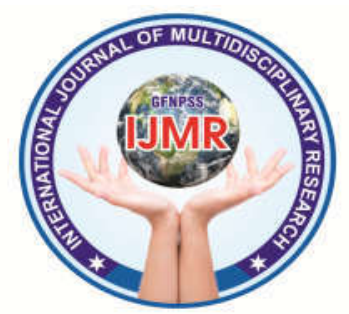

compare standard incision and comma shaped incision and its influence on post-operative complications in surgical removal of impacted third molars. J Clin Diagn Res 2013; 7:1514-1518.

05. Guven O, Keskin A, Akal UK. The incidence of cysts and tumors around impacted third molars. Int $\mathrm{J}$ Oral Maxillofac Surg 2000; 29:131-135.

06. Fernandes MJ, Ogden GR, Pitts NB, et al. Incidence of symptoms in previously symptomfree impacted lower third molars assessed in general dental practice. Br Dent J 2009; 207:E10(discussion 218-219).

07. Sidlauskas A, Trakiniene G. Effect of the lower third molars on the lower dental arch crowding. Stomatologi ja2006; 8:80-84.

08. Rakprasitkul S. Pathologic changes in the pericoronal tissues of unerupted third molars. Quintessence Int 2001; 32:633-638.

09. Ward TG. The split bone technique for removal of lower third molars. $\mathrm{Br}$ Dent J 1956;101:297-304.

10. Praveen G, Rajesh P, Neelakandan RS, Nandagopal CM. Comparison of morbidity following the removal of mandibular third molar by lingual split, surgical bur and simplified split bone technique. Indian Journal of Dental Research 2007;19(18):15- 18.

11. Kerawala CJ, Martin IC, Allan W, Williams ED. The effects of operator technique and bur design on temperature during osseous preparation for osteosynthesis selftapping screws. Oral Surg Oral Med Oral Pathol Oral Radiol Endod. 1999; 88: 145- 150 .

12. American Academy of Periodo ntology. Glossary of periodontal term. 4th ed. Chicago, 2001.

13. McArdle LW, Renton TF. Distal cervical caries in mandibular second molar: an indication for the prophylactic removal of the third molar? Br J Oral Maxillofac Surg. 2005;44:42-45. doi: 10.1016/j. Bjoms .2005 .07 .025

14. McArdle LW, Renton TF. Distal cervical caries in mandibular second molar: an indication for the prophylactic removal of the third molar? Br J Oral Maxillofac Surg. 2005;44:42-45. doi: 10.1016/j.Bjoms 2005.07.025

15. SortinoF,PedullàE,MasoliV.Thepiezo electricandrotatoryosteotomytechniqu e in impacted third molar surgery: 


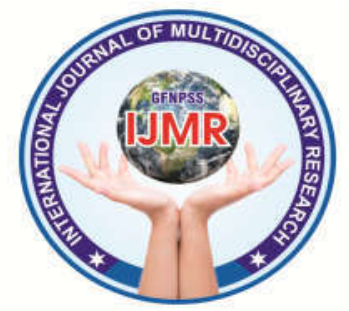

Comparison of postoperative

relationships between surgical recovery. J Oral Maxillofac Surg 66 (2008): 2444-2448.

16. SivolellaS,BerengoM,BressanE.Osteo tomyforlowerthirdmolargermectomy: Randomized prospective crossover clinical study comparing piezosurgery and conventional rotatory osteotomy. J Oral Maxillofac Surg 69 (2011):1523.

17. Goyal M, Marya K, Jhamb A, et al. Comparative evaluation of surgical outcome after removal of impacted mandibular third molars using a piezotome or a conventional handpiece: A prospective study. $\mathrm{Br} \mathrm{J}$ Oral Maxillofac Surg 50 (2012): 556561

18. RulloR,AddabboF,PapaccioG,D’Aqui noR,FestaVM.Piezoelectricdevicevs. conventional rotative instruments in impacted third molar surgery: relationships between surgical difficulty and postoperative pain with histological evaluations. J Craniomaxillofac Surg 2013; 41: e3338.

19. RulloR,AddabboF,PapaccioG,D'Aqui noR,FestaVM.Piezoelectricdevicevs. conventional rotative instruments in impacted third molar surgery: difficulty and postoperative pain with histological evaluations. J Cranio maxillofac Surg 2013; 41: e33-38.

20. Basheer S, Govind R, Daniel A, Sam G, Adarsh V, Rao A (2017) Comparative study of piezoelectric and rotary osteotomy technique for third molar impaction. J Contemp Dent Pract 18(1):60-64

21. Barone A, Marconcini S, Giacomelli $\mathrm{L}$, et al. A randomized clinical evaluation of ultrasound bone surgery versus traditional rotary instruments in lower third molar extraction. J Oral Maxillofac Surg 68 (2010): 330-336.

22. Barone A, Marconcini S, Giacomelli $\mathrm{L}$, et al. A randomized clinical evaluation of ultrasound bone surgery versus traditional rotary instruments in lower third molar extraction. J Oral Maxillofac Surg 68 (2010): 330-336.

23. Arakji H, Shokry M, Aboelsaad N. Comparison of piezosurgery and conventional rotary instruments for removal of impacted mandibular third molars: a randomized controlled clinical and radiographic trial. Int $\mathrm{J}$ Dentistry. 2016;2016:8169356 7.

24. PappalardoS,GuarnieriR.Randomized clinicalstudycomparingpiezosurgerya 


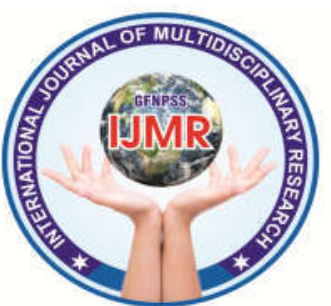

nd conventional rotatory surgery in

26. Jiang Q, Qiu Y, Yang C, Yang J, mandibular cyst enucleation. J Chen M, Zhang Z. Piezoelectric Craniomaxillofac Surg. 2014 Versus Conventional Rotary Jul;42(5):e80-5. Techniques for Impacted Third Molar

25. PappalardoS,GuarnieriR.Randomized clinicalstudycomparingpiezosurgerya nd conventional rotatory surgery in mandibular cyst enucleation. J Extraction: A Meta- analysis of Randomized Controlled Trials. Medicine (Baltimore). 2015; 94: Craniomaxillofac Surg. 2014 Jul;42(5):e80-5. 